\title{
LAT Perspectives in Detection of High Energy Cosmic Ray Electrons
}

\author{
Alexander Moiseev ${ }^{1}$, J.F. Ormes ${ }^{2}$, and Stefan Funk ${ }^{3}$ \\ ${ }^{1}$ CRESST/USRA/GSFC, NASA/GSFC, Greenbelt, MD 20771 \\ ${ }^{2}$ University of Denver, Denver, CO 80208 \\ ${ }^{3}$ Stanford Linear Accelerator Center, 2575 Sand Hill Rd., Menlo Park, CA 94025
}

\begin{abstract}
The GLAST Large Area Telescope (LAT) science objectives and capabilities in the detection of high energy electrons in the energy range from $20 \mathrm{GeV}$ to $\sim 1 \mathrm{TeV}$ are presented. LAT simulations are used to establish the event selections. It is found that maintaining the efficiency of electron detection at the level of $30 \%$ the residual hadron contamination does not exceed $2-3 \%$ of the electron flux. LAT should collect $\sim$ ten million of electrons with the energy above $20 \mathrm{GeV}$ for each year of observation. Precise spectral reconstruction with high statistics presents us with a unique opportunity to investigate several important problems such as studying galactic models of IC radiation, revealing the signatures of nearby sources such as high energy cutoff in the electron spectrum, testing the propagation model, and searching for KKDM particles decay through their contribution to the electron spectrum
\end{abstract}

Keywords: High energy cosmic ray electrons, gamma-ray telescope

PACS: $95.55 . \mathrm{Ka}, \mathbf{9 5 . 5 5 . V j}$

An accompanying paper [1] discusses the science topics that can be studied using precision measurements of the cosmic ray electron spectrum arriving at Earth. Most of the published results have been obtained in balloonborne experiments ([2] and references therein) and have very limited statistics, which leave ambiguity in their interpretation. We explored the capability of the GLAST Large Area Telescope (LAT) to detect cosmic ray electrons in the $20 \mathrm{GeV}$ to $\sim 1 \mathrm{TeV}$ energy range, and have found that the data can be obtained reliably and the background removed effectively.

Being a gamma-ray telescope, LAT is already an excellent detector of electrons. The main problem is to separate the electrons from all other species, mainly from protons; proton-electron separation power above $\sim 20$ $\mathrm{GeV}$ is needed to be $10^{3}-10^{4}$ in order to have not more than $10 \%$ proton contamination in measured electron flux. It is assumed that the gamma-rays can be effectively identified and removed from the electron data set by the LAT Anti-Coincidence Detector [3].

LAT includes a configurable trigger mode that sends all events with energy $>10-20 \mathrm{GeV}$ in calorimeter to the ground for further analysis. Our data will be in the set with this trigger. We should not have any problem with albedo and geomagnetic variation at such a high energy. We developed a set of analysis cuts that select the electrons. These cuts are applied to the ntuple variables containing simulated data from LAT. The basic approach is to utilize the difference between electron-initiated and hadron-initiated event patterns in LAT by the following selections:

- remove events which passed the LAT without interaction or interacted too deep in the LAT calorimeter. This removes $\sim 75 \%$ of all hadrons;

- remove events which have scattered hit calorimeter crystals at some distance from shower axis, using the fact that a lepton-initiated shower in the calorimeter is much more compact (dense) than for most hadron-initiated events;

- remove events with large energy deposition in ACD tiles, using the fact that hadron-initiated events have (on average) larger number of backward moving charged particles which create excessive hits in the ACD;

- remove events with larger asymmetry of the shower shape in the calorimeter - electron-initiated showers have much smaller shape fluctuation; 


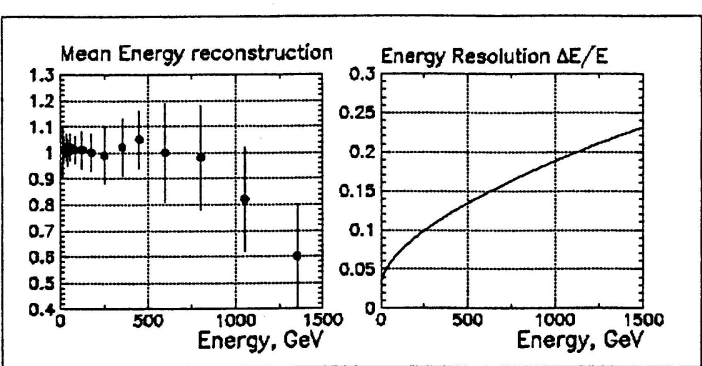

FIGURE 1. Left panel - Mean value of reconstructed energy; Right panel - energy resolution of the events passed electron selections

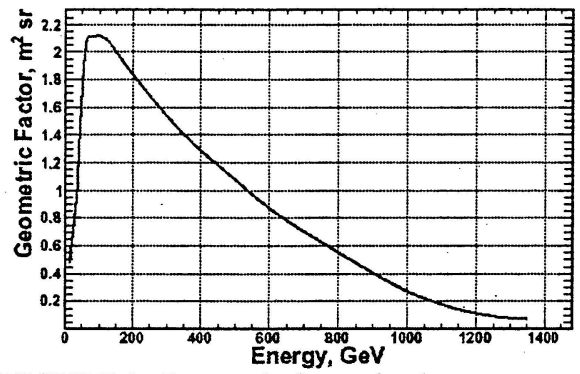

FIGURE 2. Geometric factor for the events passed electron selections

- accept as electrons the events that start their shower multiplication in the tracker - this is a powerful tool, but it also removes a large fraction of the electrons;

- select events with a path in the calorimeter projected from the tracker to be larger than $8 \mathrm{X}_{0}$ - this improves the energy resolution.

These cuts were applied to the charged particle background used by the LAT collaboration to simulate the flight data [4]. The resulting efficiency for keeping electrons is $\sim 30 \%$ with a residual proton contamination of $\sim 3 \%$ of after applying all listed cuts. A very important issue is the energy reconstruction. The mean value of the ratio of reconstructed energy and the Monte Carlo input energy is shown in the left panel of Fig.1, and the energy resolution is shown in right panel. The energy underestimation above $\sim 700 \mathrm{GeV}$ can be corrected in the analysis, extending the results up to $1 \mathrm{TeV}$. The resulting geometric factor for electrons is shown in Fig.2.

We tested the spectral reconstruction with these cuts. First we simulated an incident "mythological" electron flux postulated to originate from a single burst-like source, $2 \times 10^{5}$ years old, distanced at 100 parsec. The electrons propagate to Earth through a scattering medium with an assumed diffusion $D_{0}=D\left(E / E_{0}\right)^{0.6}$ with $D_{0}=10^{28} \mathrm{~cm} / \mathrm{s}^{2}$ and with an expected energy cutoff at $\sim 1.2 \mathrm{TeV}$ ([5], also [2], [6], [7]). The simulated results of the flux reconstruction are shown in Fig.3. The spectrum is well reconstructed up to $\sim 1 \mathrm{TeV}$ (a result that can be compared with currently available measurements from [2] which is shown as Fig.1 in [1]). There is an indication of the spectral cutoff in the reconstructed spectrum, but further improvement of the reconstruction is needed at high energy.

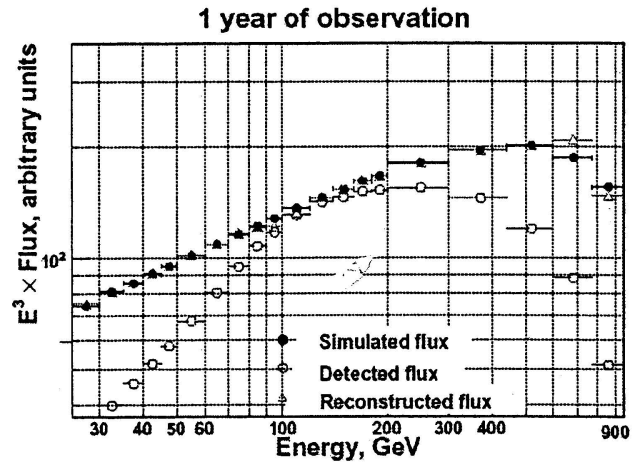

FIGURE 3. Simulations of the electron spectrum

\section{SUMMARY}

Utilizing the selections we have developed, the electron spectrum can be well reconstructed with 1 year's LAT data. We expect to detect $\sim 10^{7}$ electrons/yr above $20 \mathrm{GeV}, 4 \times 10^{5}$ electrons/yr above $100 \mathrm{GeV}$, and 2,500 electrons/yr above $500 \mathrm{GeV}$ assuming a steep power law electron spectrum with power law index -3.3. Further improvement can be expected once we employ the ClassificationTree technique. In future we should be able to extend the analysis to lower energies and improve the statistical precision at high energy.

\section{REFERENCES}

1. Ormes, J.F., and Moiseev, A.A., These Proceedings

2. Kobayashi T., et al., ApJ 601 (2004), 340

3. Moiseev, A., et al., Astroparticle Physics, in press

4. Ormes, J.F., et al. These Proceedings

5. Atoyan, A.M., Aharonian, F.A., and Volk, H.J., Physical Review D, 52 (1995), 3265

6. Nishimura, J., et al., ApJ 238 (1980), 394

7. Aharonian, F.A., Atoyan, A.M., and Volk, H.J., A\&A 294 (1995), L41 